\title{
Effective component of Salvia miltiorrhiza in promoting cardiomyogenic differentiation of human placenta-derived mesenchymal stem cells
}

\author{
KUN LI ${ }^{1}$, JIEQIONG SONG ${ }^{1}$, QIAN ZHAO ${ }^{1}$, BO WANG ${ }^{1}$, YUNLI ZHANG ${ }^{1}$, \\ XUEZHE WANG ${ }^{1}$, TIANTIAN TANG ${ }^{1}$ and SHIZHENG LI $^{2}$ \\ Departments of ${ }^{1}$ Clinical Laboratory and ${ }^{2}$ General Surgery, \\ The First Affiliated Hospital of Jinzhou Medical University, Jinzhou, Liaoning 121001, P.R. China
}

Received April 13, 2017; Accepted November 23, 2017

DOI: 10.3892/ijmm.2017.3293

\begin{abstract}
Our previous study indicated that Salvia miltiorrhiza (SM) induced human placenta-derived mesenchymal stem cells (hPDMSCs) to differentiate into cardiomyocytes, however, the effective component of SM in promoting cardiomyogenic differentiation remained to be elucidated. In the present study, the most commonly examined components of SM, including danshensu, salvianolic acid B, protocatechuic aldehyde, tanshinone I (TS I), TS IIA and cryptotanshinone, were used to determine the effective components of SM in promoting cardiomyogenic differentiation. The above components of SM slowed cell growth rate and altered cell morphology with a spindle or irregular shape to different degrees. The cells treated with the above components of SM showed increasing of cardiac protein expression to differing degrees, including GATA-binding protein 4 , atrial natriuretic factor, $\alpha$-sarcomeric actin and cardiac troponin-I. Among the components of SM, TS IIA induced the most marked effects. In addition, the above components of SM increased the expression of phosphorylated glycogen synthase kinase-3 $\beta$, but decreased the expression of $\beta$-catenin to different degrees, with TS IIA also having the
\end{abstract}

Correspondence to: Dr Shizheng Li, Department of General Surgery, The First Affiliated Hospital of Jinzhou Medical University, 2 Renmin Road, Jinzhou, Liaoning 121001, P.R. China

E-mail: 1szlklym@163.com

Abbreviations: hPDMSCs, human placenta-derived mesenchymal stem cells; SM, Salvia miltiorrhiza; DSS, danshensu; SA B, salvianolic acid B; PCAD, protocatechuic aldehyde; TS I, tanshinone I; TS IIA, tanshinone IIA; CTS, cryptotanshinone; ANF, atrial natriuretic factor; $\alpha$-SCA, $\alpha$-sarcomeric actin; cTnI, cardiac troponin-I; p-GSK-3 $\beta$, phosphorylated glycogen synthase kinase-3 $\beta$; BMSCs, bone marrow mesenchymal stem cells; PBS, phosphate buffer solution; PFA, paraformaldehyde; FITC, fluorescence isothiocyanate

Key words: Salvia miltiorrhiza, effective components, human placenta-derived mesenchymal stem cells, cardiomyocytes most marked effects. In conclusion, the results of the present study suggested that TS IIA was the most effective active component of SM in inducing hPDMSCs to differentiate into cardiomyocytes, and that Wnt/ $\beta$-catenin signaling was important in the process of TS IIA promoting cardiomyogenic differentiation.

\section{Introduction}

Traditional medical and surgical therapies have been successful in treating several cardiovascular diseases, however, a number of patients suffer from heart failure due to the irreversible loss of cardiomyocytes caused by myocardial ischemia. Although increasing evidence indicates that cardiac progenitor cells may be involved in the process of myocardial regeneration in patients with heart failure (1-3), the degree of this potential remains controversial (4-7). Stem cell biology has provided therapeutic approaches for replacing non-functional cardiac tissue by cell transplantation. In principle, autologous bone marrow mesenchymal stem cells (BMSCs) are the ideal cells for use in transplantation. However, cardiovascular disease occurs more often in middle-aged and elderly individuals, and the number and function of BMSCs are depressed in these patient groups (8). This suggests limitations in the utilization of autologous stem cells for patients with ischemic cardiomyopathy. Human placenta-derived mesenchymal stem cells (hPDMSCs) possess the ability of multi-directional differentiation (9); in addition, hPDMSCs are widely sourced and easier to isolate, which makes them an attractive alternative stem cell source, particularly for transplantation purposes.

The pre-differentiation of stem cells towards a defined cardiac lineage prior to transplantation may be more advantageous than transplanting uncommitted stem cells, which may undergo unanticipated differentiation. Several methods have been used to promote the cardiomyogenic differentiation of stem cells, including co-culturing techniques $(10,11)$, demethylating agent treatment (12), and specific gene insertions (13). However, the majority of these methods are unlikely to be clinically applied due to their low efficacy and potentially harmful effects (14). Salvia miltiorrhiza (SM), a well-known traditional Chinese medicine, is widely used in China and 
neighboring countries to treat patients suffering from ischemic heart disease with minimal side effects. Our previous study (15) indicated that SM induced hPDMSCs to differentiate into cardiomyocytes, and this effect was superior to that of the co-culture method. The pre-differentiation of stem cells towards a defined cardiac lineage with SM prior to transplantation is likely to be clinically applicable. However, the effective components of SM in promoting cardiomyogenic differentiation remain to be fully elucidated. Determining the effective component of SM in promoting cardiomyogenic differentiation may contribute to investigations aimed at enhancing the efficiency of cardiomyogenic differentiation and myocardial regeneration. The present study was performed to investigate the effective component of SM in promoting cardiomyogenic differentiation and its possible mechanism.

\section{Materials and methods}

hPDMSC isolation and identification. Placentas at term (38-40 weeks gestation; $n=10$ ) were obtained from healthy donor mothers following the provision of informed consent and according to the procedures of the institutional review board. The present study was approved by the Ethics Committee of The First Affiliated Hospital of Jinzhou Medical University (Jinzhou, China). Briefly, the cells were isolated using a tissue culture method (15) and cultured in $5 \% \mathrm{CO}_{2}$ at $37^{\circ} \mathrm{C}$, following which the placenta-derived cells at the fourth passage were treated with $0.25 \%$ trypsin-EDTA, harvested, and washed twice with phosphate-buffered solution (PBS). The cells were incubated on ice for $20 \mathrm{~min}$ with pre-diluted PE-labeled mouse anti-human antibodies CD13 (cat. no. 560998), CD73 (cat. no. 561014), CD90 (cat. no. 561970), CD166 (cat. no. 559263), HLA-DR (cat. no. 555561) and FITC-labeled mouse anti-human antibodies CD14 (cat. no. 555397) (all from BD Biosciences, San Jose, CA, USA), CD29 (cat. no. 11-0299-41; eBiosiences, San Diego, CA, USA), CD31 (cat. no. 560984), CD44 (cat. no. 560977), CD45 (cat. no. 561865), CD105 (cat. no. 561443) and HLA-ABC (cat. no. 557348). Control groups were incubated with FITC-(cat. no. 555786) and PE-conjugated mouse anti-human IgG (cat. no. 555787) (all from BD Biosciences). The labeled cells were analyzed using flow cytometry (BD FACSCanto II; Becton-Dickinson Co., Franklin Lakes, NJ, USA).

Cardiomyogenic differentiation. Briefly, the proliferation of hPDMSCs was measured using an MTS assay following treatment with SM and its most commonly examined components, including danshensu (DSS), salvianolic acid B (SA B), protocatechuic aldehyde (PCAD), tanshinone I (TS I), tanshinone IIA (TS IIA) and cryptotanshinone (CTS), at different concentrations for 4 days. As concentrations of $\mathrm{SM}<4 \mathrm{mg} / 1$, DSS $<10 \mathrm{mg} / 1$, SA B $<10 \mathrm{mg} / 1$, PCAD $<0.5 \mathrm{mg} / 1$, TS I $<1 \mathrm{mg} / 1$, TS IIA $<0.1 \mathrm{mg} / 1$ and CTS $<0.1 \mathrm{mg} / 1 \mathrm{had}$ minimal effect on cell proliferation (Fig. 2A), 4 mg/l SM, $10 \mathrm{mg} / 1 \mathrm{DSS}, 10 \mathrm{mg} / \mathrm{l}$ SA B, $0.5 \mathrm{mg} / 1 \mathrm{PCAD}, 1 \mathrm{mg} / 1 \mathrm{TS}$ I, $0.1 \mathrm{mg} / 1 \mathrm{TS}$ IIA and $0.1 \mathrm{mg} / \mathrm{l} \mathrm{CTS}$ concentrations were preferred to enable superior assessment of the effective components of SM in promoting cardiomyogenic differentiation in experiments. The hPDMSCs were inoculated in 96-well plates, 24 -well plates and $10-\mathrm{cm}$ culture dishes at a density of $1 \times 10^{3}$ cells $/ \mathrm{cm}^{2}$ and cultured in
$5 \% \mathrm{CO}_{2}$ at $37^{\circ} \mathrm{C}$. The following day, the cells were respectively treated with $4 \mathrm{mg} / 1 \mathrm{SM}, 10 \mathrm{mg} / 1 \mathrm{DSS}, 10 \mathrm{mg} / 1 \mathrm{SA} \mathrm{B}, 0.5 \mathrm{mg} / 1$ PCAD, $1 \mathrm{mg} / 1 \mathrm{TS}$ I, $0.1 \mathrm{mg} / \mathrm{l} \mathrm{TS}$ IIA and $0.1 \mathrm{mg} / 1 \mathrm{~T}$ CTS in complete medium containing Dulbecco's modified Eagle's medium supplemented with $10 \%$ fetal bovine serum (Gibco, Gaithersburg, MD, USA), $1 \mathrm{mmol} / 1 \mathrm{~L}$-glutamine, $0.1 \mathrm{mmol} / \mathrm{l}$, $\beta$-mercaptoethanol and $1 \%$ non-essential amino acids for the induction of cardiomyogenic differentiation. The aforementioned medium was replaced every 4 days. The morphologic characteristics of the cells were analyzed under a microscope (Leica DMI1; Leica Microsystems Inc; Mannheim, Germany) every day. As the absorbance values in an MTS assay can indirectly reflect the number of living cells, with the absorbance values being positively associated with the number of living cells, cell proliferation was measured using the MTS assay every 4 days. The experiment was terminated at day 20 .

Immunocytochemistry. The cells were fixed in PBS containing 4\% paraformaldehyde (PFA) for $20 \mathrm{~min}$, permeabilized in PBS containing $0.2 \%$ Triton X-100 for $10 \mathrm{~min}$, and blocked in a serum-free blocking solution for $5 \mathrm{~min}$ at room temperature. The cells were then incubated with primary antibody against a-SCA (1:100 dilution; cat. no. ab9465; Abcam, Cambridge, UK) overnight at $4^{\circ} \mathrm{C}$. Following extensive washing with PBS, the cells were incubated with HRP-conjugated goat anti-rabbit IgG (1:100 dilution; cat. no. ab6721; Abcam). Finally, the nuclei were stained by incubation with hematoxylin. The results were analyzed under a microscope (Leica DMI1; Leica Microsystems Inc.).

Immunofluorescence. The cells were washed three times with PBS and fixed in PBS containing 4\% PFA for $30 \mathrm{~min}$. The fixed cells were washed three times with PBS and permeabilized in PBS containing $0.2 \%$ Triton X-100 for $10 \mathrm{~min}$. The cells were then washed three times with PBS and blocked in a serum-free blocking solution for $5 \mathrm{~min}$ at room temperature. The blocked cells were incubated with cardiac troponin-I (cTnI) primary antibody (1:50 dilution; cat. no. ab47003; Abcam) overnight at $4^{\circ} \mathrm{C}$. Following extensive washing with PBS, the cells were incubated with FITC-conjugated goat anti-rabbit IgG (1:50 dilution; cat. no. ab6717; Abcam) for $1 \mathrm{~h}$ at room temperature. Following extensive washing with PBS, the nuclei were stained by incubation with propidium iodide (PI). The results were analyzed using a fluorescence microscope. The differentiation ratio of cardiomyocytes was calculated as the fraction of cTnI-positive cells in total cells, which were in a counterpart visual field. The rate was calculated as the average of $>10$ separate fields.

Western blot analysis. The cells were washed twice with PBS and then collected. Total proteins were extracted with cell lysis buffer (cat. no. P0013b; Beyotime) according to the manufacturer's protocol. The protein quantity was determined by BCA assay (BioTek, Winooski, VT, USA). Equal quantities of protein $(30 \mu \mathrm{g})$ were respectively separated with sodium dodecyl sulfate-polyacrylamide gel electrophoresis on 8 or $10 \%$ gel and then transferred onto polyvinylidene difluoride membranes. The membranes were blocked with $5 \%$ non-fat milk for $1 \mathrm{~h}$ and then incubated overnight at $4^{\circ} \mathrm{C}$ with the following primary antibodies: GAPDH (1:5,000 dilution; cat. 

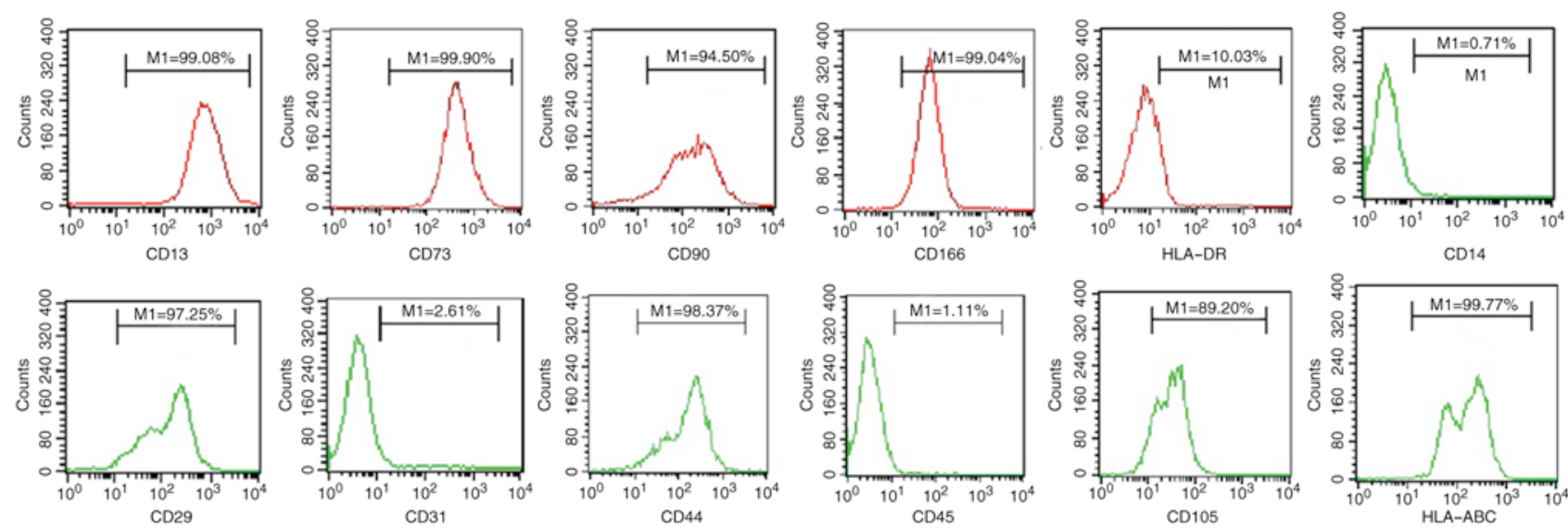

Figure 1. Identification of human placenta-derived mesenchymal stem cells. The placenta-derived cells at passage 4 were analyzed using flow cytometry. The results showed that the cells were positive for CD13, CD29, CD44, CD73, CD90, CD105, CD166 and HLA-ABC, but negative for CD14, CD31, CD45 and HLA-DR.

no. ab9485), GATA-binding protein 4 (GATA4; 1:1,000 dilution; cat. no. ab84593), atrial natriuretic factor (ANF; 1:1,000 dilution; cat. no. ab14348), cTnI (1:1,000 dilution; cat. no. ab47003), glycogen synthase kinase-3 $\beta$ (GSK-3 $\beta ; 1: 10,000$ dilution; cat. no. ab32391), phosphorylated (p-)GSK-3 $\beta$ (1:10,000 dilution; cat. no. ab75814) or $\beta$-catenin (1:10,000 dilution; cat. no. ab10000), all from Abcam. This was followed by incubation with HRP-conjugated goat anti-rabbit IgG (1:5,000 dilution; cat. no. ab6721; Abcam) for $2 \mathrm{~h}$ at room temperature. Antibody detection was performed using a chemiluminescence detection kit (Omega Lum G; Aplegen Inc., Pleasanton CA, USA).

Statistical analysis. Each experiment was repeated at least three times. All data are presented as the mean \pm standard deviation. A one-way analysis of variance was used for comparisons among the groups. Statistical analysis was performed using SPSS 13.0 software. $\mathrm{P}<0.05$ was considered to indicate a statistically significant difference.

\section{Results}

Characterization of human placenta-derived cells. The placenta-derived cells grew to form colonies and exhibited an adherent fibroblastoid appearance. The results of the flow cytometric analysis demonstrated that the placenta-derived cells were positive for CD13, CD29, CD44, CD73, CD90, CD105, CD166 and HLA-ABC, and negative for CD14, CD31, CD45 and HLA-DR (Fig. 1). The surface markers of these placenta-derived cells were exactly the same as those of previously reported bone marrow- and cord blood-derived mesodermal cells (16).

Effects of SM and its active components on cell proliferation and cell morphology. To better assess whether SM and its active components inhibited hPDMSC proliferation and stimulated cell differentiation, cell proliferation was evaluated using an MTS assay and cell morphological characteristics were analyzed using microscopy. The cells in the negative control group maintained an increased growth rate and maintained fibroblast-like morphology. However, the cells treated with SM and its active components exhibited a slower growth rate, with certain cells beginning to exhibit altered morphology. These cells gradually increased in size to form a stick-like appearance. On day 20, the cells became enlarged and exhibited a number of branches connecting with adjoining cells (Fig. 2A). Among the SM components, TS IIA had the most marked effect on the cells (Figs. 2B and 3).

Effects of SM and its active components on the cardiomyogenic differentiation of hPDMSCs. In order to corroborate the cardiomyogenic differentiation of hPDMSCs, immunohistochemistry was performed to detect $\alpha$-SCA, and western blot analysis was performed to detect the protein expression levels of GATA4, ANF and cTnI. The immunohistochemistry revealed that some of the treated cells stained positive for $\alpha$-SCA, and there were more $\alpha$-SCA-positive cells in the TS IIA treatment group (Fig. 4A). Compared with the negative control group, the protein expression levels of GATA4, ANF and cTnI were increased in the treatment groups, however, the protein expression levels of GATA4, ANF and cTnI were marginally higher in the cells treated with TS IIA (Fig. 4B and C).

Effects of Tan IIA on Wnt/ $\beta$-catenin signaling. In order to determine whether $\mathrm{Wnt} / \beta$-catenin signaling was important in $\mathrm{SM}$ and its active components in promoting the cardiomyogenic differentiation of hPDMSCs, the protein expression levels of GSK-3 $\beta$, p-GSK-3 $\beta$ and $\beta$-catenin were examined. The results showed that the expression levels of GSK-3 $\beta$ and p-GSK-3 $\beta$ were increased to different degrees, whereas the expression of $\beta$-catenin was decreased to different degrees in the treatment groups. In addition, the ratios of $\mathrm{p}-\mathrm{GSK}-3 \beta$ to GSK-3 $\beta$ were increased to different degrees, particularly in the TS IIA treatment group (Fig. 4B and C).

Effects of SM and its active components on cardiomyogenic differentiation rate. For further analysis of the effective components of SM in promoting cardiomyogenic differentiation, immunofluorescence was performed to detect cardiomyogenic differentiation rate. cTnI was not expressed in the control group. The expression levels of cTnI in the treatment groups were different, with the cardiomyogenic induction rate of the TS IIA being marginally higher (Fig. 5). 
A
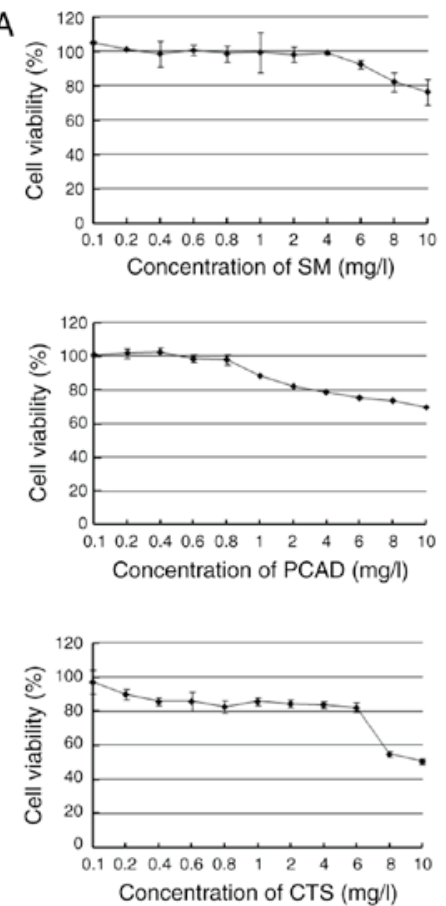
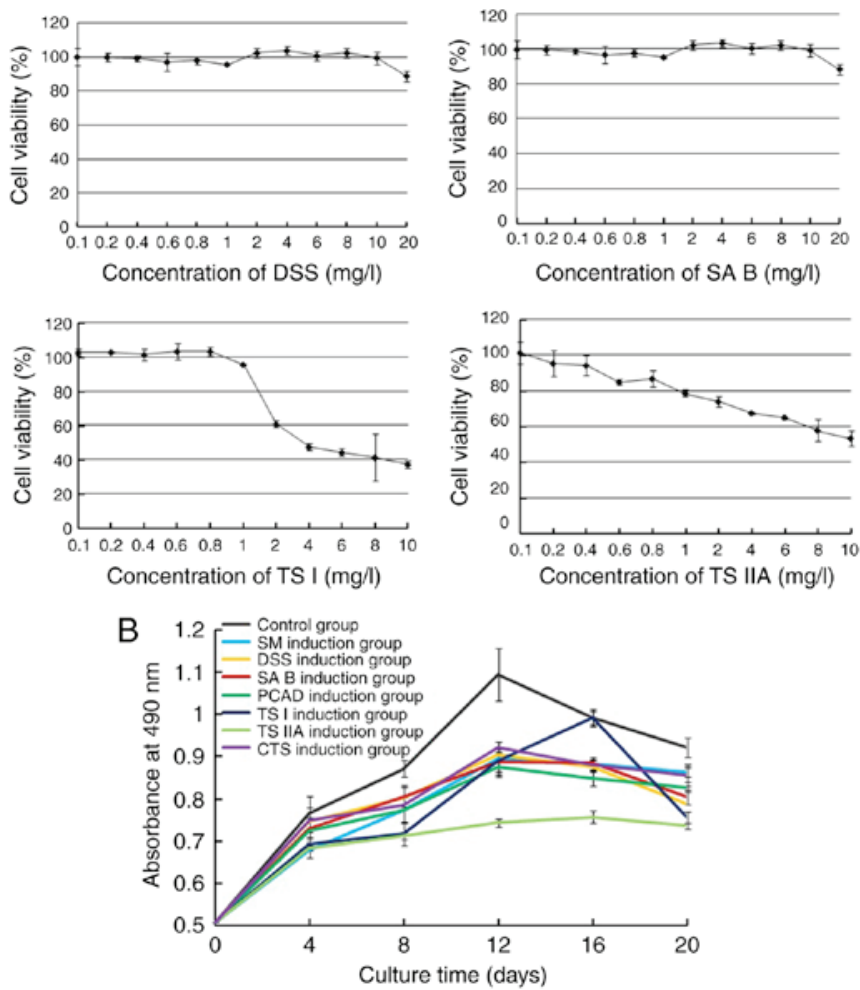

Figure 2. Effects of SM and its active components on the proliferation of hPDMSCs. (A) Cytotoxicity of SM and its active components against hPDMSCs. Concentrations of SM $<4 \mathrm{mg} / 1$, DSS $<10 \mathrm{mg} / 1$, SA B $<10 \mathrm{mg} / 1$, PCAD $<0.5 \mathrm{mg} / 1$, TS I $<1 \mathrm{mg} / 1$, TS IIA $<0.1 \mathrm{mg} / 1 \mathrm{and} \mathrm{CTS}<0.1 \mathrm{mg} / 1$ had minimal effect on cell proliferation. (B) Effects of SM and its active components on cell proliferation. Cells in the control group maintained a higher growth rate, however, treated cells exhibited significantly reduced growth rate. TS IIA had the most marked effect. Data are presented as the mean \pm standard deviation of three independent experiments. hPDMSCs, human placenta-derived mesenchymal stem cells; SM, Salvia miltiorrhiza; DSS, danshensu; SA B, salvianolic acid B; PCAD, protocatechuic aldehyde; TS I, tanshinone I; TS IIA, tanshinone IIA; CTS, cryptotanshinone.
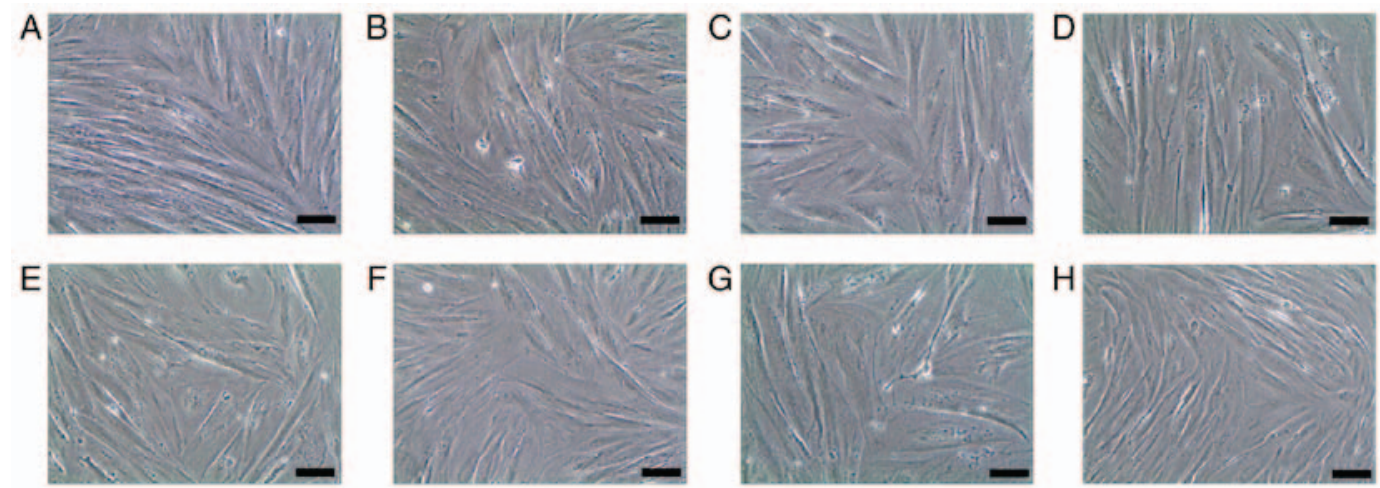

Figure 3. Effects of SM and its active components on cell morphology. (A) Negative control, cells maintained their fibroblast-like morphology; (B) SM induction group; (C) danshensu induction group; (D) salvianolic acid B induction group; (E) protocatechuic aldehyde induction group; (F) TS I induction group; (G) TS IIA induction group; (H) cryptotanshinone induction group. A number of cells became enlarged and exhibited a number of branches connecting with adjoining cells. TS IIA had the most marked effect. Scale bar, $20 \mu \mathrm{m}$. TS tanshinone; SM, Salvia miltiorrhiza.

\section{Discussion}

The recovery of cardiac performance following cellular transplantation in experimental models has been partly attributed to the transdifferentiation of stem cells leading to de novo formation of cardiomyocytes $(17,18)$. However, the types and characteristics of these stem cells remain poorly defined and widely different transdifferentiation efficiency has been reported (19). The pre-differentiation of stem cells into cardiomyocytes may enhance their abilities to survive and engraft as cardiomyocytes following myocardial transplantation.
In addition, the transplantation of cells with pre-defined myocardial characteristics may circumvent the challenges of transdifferentiation and cell fusion in the generation of cardiomyocytes by undifferentiated stem cells in the regenerating myocardium. At present, several methods have been used to promote the cardiomyogenic differentiation of stem cells, including co-culturing techniques $(10,11)$, treatment with demethylating agents (12), and specific gene insertions (13). However, the majority of these methods are unlikely to be clinically applied due to their low efficacy and potentially harmful effects (14). 

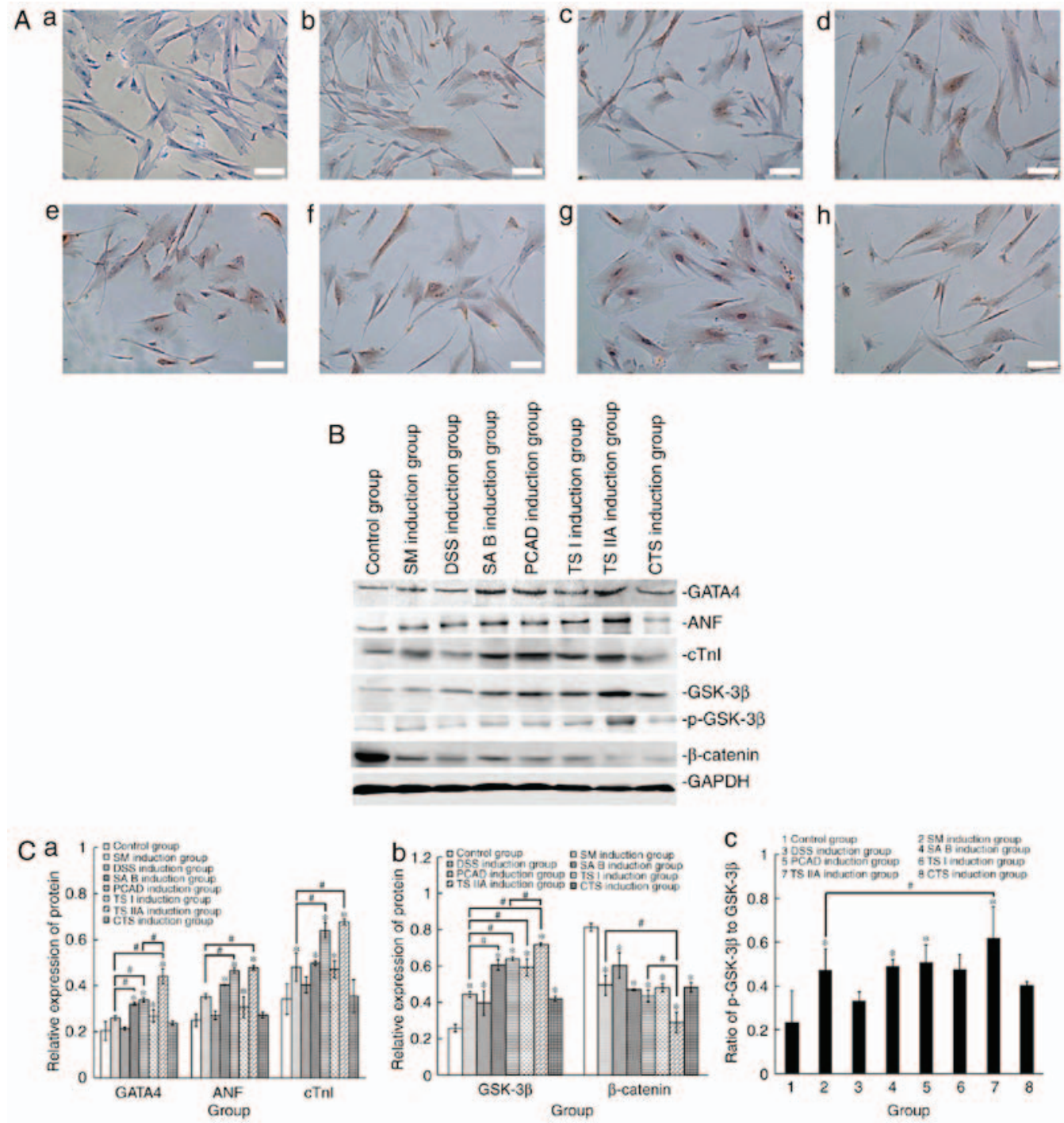

Figure 4. Effects of SM and its active components on cardiomyogenic differentiation of human placenta-derived mesenchymal stem cells. (A) Expression of $\alpha$-SCA; (a) negative control, cells stained negative for $\alpha$-SCA; (b) SM induction group; (c) DSS induction group; (d) SA B induction group; (e) PCAD induction group; (f) TS I induction group; (g) TS IIA induction group; (h) CTS induction group, a number of cells stained positively for $\alpha$-SCA, with more $\alpha$-SCA positive cells in TS IIA induction group. Scale bar, $20 \mu \mathrm{m}$. (B) Results of the western blot analysis of GATA4, ANF, cTnI, GSK-3 $\beta$, p-GSK-3 $\beta$ and $\beta$-catenin. (C) Relative protein expression levels of (a) GATA4, ANF and cTnI, and (b) GSK-3 $\beta$ and $\beta$-catenin. (c) Ratios of p-GSK-3 $\beta$ to GSK-3 $\beta$. Data are presented as the mean \pm standard deviation of three independent experiments. ${ }^{*} \mathrm{P}<0.05$ compared with the control group; ${ }^{\text {" }} \mathrm{P}<0.05$ between the two groups. SM, Salvia miltiorrhiza; DSS, danshensu; SA B, salvianolic acid B; PCAD, protocatechuic aldehyde; TS I, tanshinone I; TS IIA, tanshinone IIA; CTS, cryptotanshinone; ANF, atrial natriuretic factor; $\alpha$-SCA, $\alpha$-sarcomeric actin; cTnI, cardiac troponin-I; GSK-3 $\beta$, glycogen synthase kinase-3 $\beta$; p-GSK-3 $\beta$, phosphorylated GSK-3ß.

SM is an important Chinese natural herb used in the treatment of several diseases, particularly ischemic cardiovascular diseases. Previous studies on its potential cardioprotective effects revealed effects in dilating coronary vessels, improving the microcirculation of ischemic region, inhibiting platelet aggregation and inflammatory responses, lowering blood lipids, and inhibiting the development of arteriosclerosis. Our previous study (15) indicated that SM induced hPDMSCs to differentiate into cardiomyocytes and the effect was superior to that of the co-culture method. As is already known, the active components of SM are generally divided into two major groups, water-soluble phenolic compounds and lipophilic diterpene quinines (20). According to the Chinese Pharmacopeia (21), the content of SA B in water-soluble phenolic compounds, and the content of TS I, TS IIA and CTS in lipophilic diterpene quinines are important indicators in assessing SM quality; in dried products of SM, the recommended content of SA B is $\geq 3.0 \%$, and those of TS I, TS IIA and CTS are $\geq 0.25 \%$ each. According to the active components of SM produced by 19 major production bases of SM in China, in which the average content of DSS was $7.15 \%$ and the average content of PCAD was $1.22 \% \mathrm{Li}$ et al suggested that the Chinese Pharmacopoeia incorporate DSS and PCAD as indicators of SM quality assessment (22). Our previous studies did not determine the effective component of SM in promoting cardiomyogenic differentiation. However, determining the effective component of SM in promoting cardiomyogenic differentiation may contribute to enhancing the efficiency of cardiomyogenic differentiation and myocardial regeneration. For improved assessment of the effective components of SM in promoting cardiomyogenic differentiation, the present study used non-toxic doses of SM and its active components for 

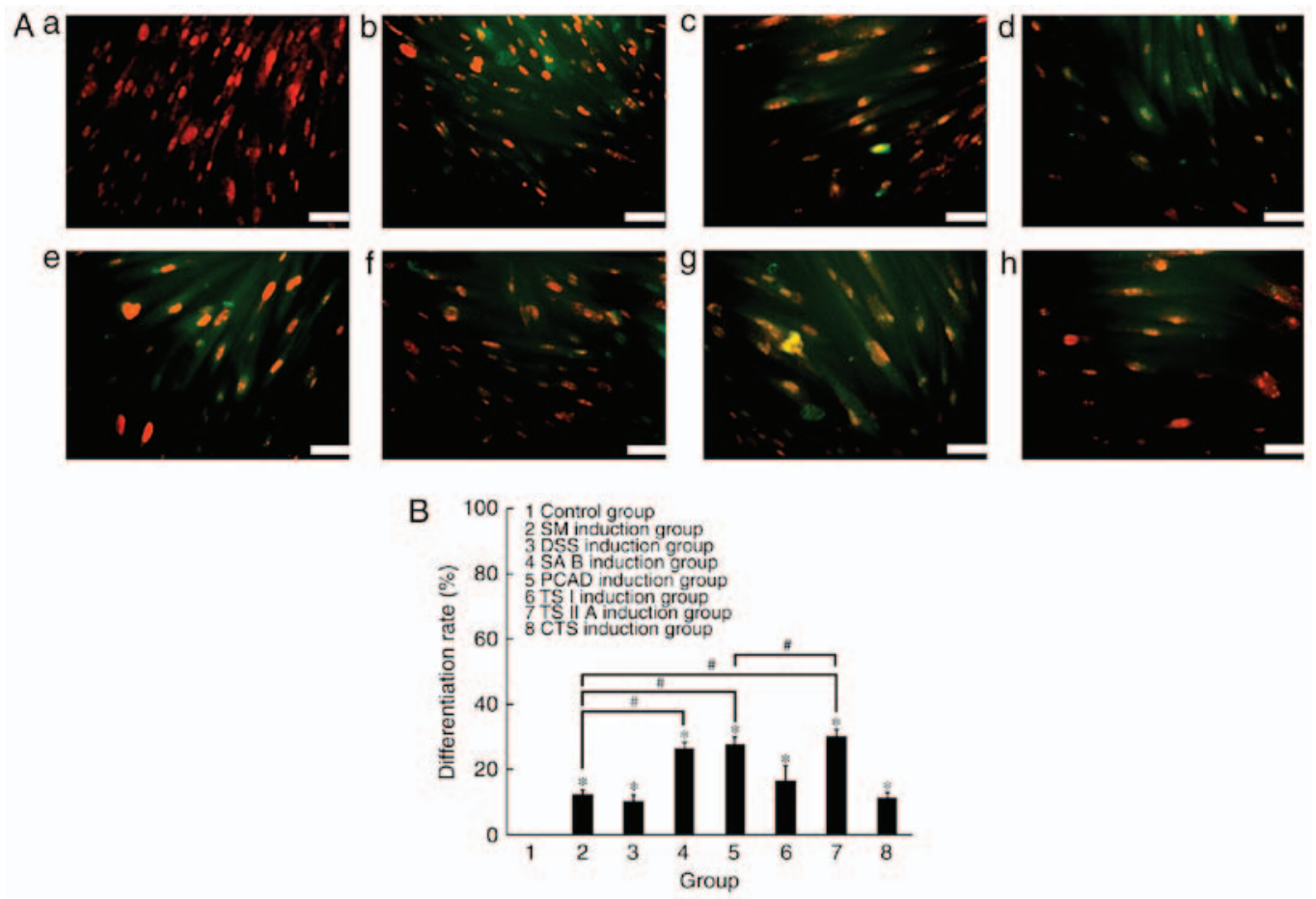

Figure 5. Effects of SM and its active components on the cardiomyogenic differentiation rate of human placenta-derived mesenchymal stem cells. (A) Expression of cTnI; (a) negative control cells stained negative for cTnI; (b) SM induction group; (c) DSS induction group; (d) SA B induction group; (e) PCAD induction group; (f) TS I induction group; (g) TS IIA induction group; (h) CTS induction group, positive staining for cTnI was observed. The nuclei were stained with propidium iodide (red) and cTnI antibody was stained using fluorescence isothiocyanate (green). Scale bar, $20 \mu \mathrm{m}$. (B) Cardiomyogenic differentiation rate. The cardiomyogenic induction rate of TS IIA was marginally higher, compared with that of SM and its the other active components. "P<0.05 compared with the control group; ${ }^{\#} \mathrm{P}<0.05$ between the two groups. SM, Salvia miltiorrhiza; DSS, danshensu; SA B, salvianolic acid B; PCAD, protocatechuic aldehyde; TS I, tanshinone I; TS IIA, tanshinone IIA; CTS, cryptotanshinone.

experiments. As SM is a complex of several components, its non-toxic dose is closely associated with the content of each monomer in SM and the non-toxic dose of each monomer. The non-toxic doses of SM and its main active components used in the present study were different (SM, $4 \mathrm{mg} / \mathrm{l}$; DSS, $10 \mathrm{mg} / \mathrm{l}$; SA B, 10 mg/l; PCAD, 0.5 mg/l; TS I, 1 mg/l; TS IIA, $0.1 \mathrm{mg} / 1$ and CTS, $0.1 \mathrm{mg} / \mathrm{l}$ ), and the statements above may explain why the non-toxic dose of SM may be higher or lower than the non-toxic dose of each monomer component. However, this does not mean that a high concentration of a non-toxic monomer has a superior effect on cardiomyogenic differentiation. The results of the present study demonstrated that SM and its effective components reduced the cell growth rate to different degrees and altered cell morphology to produce a spindle or irregular shape. The cells treated with SM and its effective components stained positively for $\alpha$-SCA and showed increased cardiac protein expression of GATA4, ANF and cTnI to different degrees. Among the treatment groups, the effect of TS IIA was the most marked, and the effect of DSS was marginally lower, compared with that of SM. These results suggested that TS IIA was the most effective active component of SM in inducing hPDMSCs to differentiate into cardiomyocytes. As one of the effective active ingredients of SM, TS IIA is currently used in China and other neighboring countries to treat ischemic heart disease in the clinic (23). It has been shown to exert beneficial effects on the cardiovascular system with minimal side effects, including improving the microcirculation of ischemic regions (24), inhibiting platelet aggregation (25) and inflammatory responses (26), lowering blood lipids (27), and inhibiting the development of arteriosclerosis (28). Therefore, pretreatment of these multipotent cells by TS IIA can be used prior to transplantation investigations.

Previous studies have shown that Wnt/ $\beta$-catenin signaling is important in the cardiomyocyte differentiation of MSCs. GSK-3 $\beta$ is a central component of the canonical Wnt pathway and negatively regulates $\beta$-catenin through phosphorylation-dependent proteolytic degradation (29). The downregulation of $\beta$-catenin is important in mediating the effect of GSK-3 $\beta$ on the differentiation of MSCs into cardiomyocytes (30). The results of the present study demonstrated that the expression of GSK-3 $\beta$ and $p-G S K-3 \beta$ increased to different degrees, whereas that of $\beta$-catenin decreased to different degrees in the treatment groups. In addition, the ratio of $p-G S K-3 \beta$ to GSK-3 $\beta$ increased to different degrees, and was particularly increased the TS IIA treatment group. Therefore, it was concluded that the Wnt/ $\beta$-catenin signaling pathway was inhibited in the hPDMSC differentiation process.

In conclusion, TS IIA was identified as the most effective active component of the most commonly investigated compounds of SM, including DSS, SA B, PCAD, TS I, TS IIA and CTS, in inducing hPDMSCs to differentiate into cardiomyocytes. In the hPDMSC differentiation process, the Wnt/ $\beta$-catenin signaling pathway was inhibited. The pretreatment of multipotent cells by TS IIA can be used prior to transplantation investigations to ensure that the differentiation process is directed towards the cardiomyogenic lineage in the in vivo environment. 


\section{Acknowledgements}

This study was supported by the National Natural Science Foundation (grant no. 81303255), the Natural Science Foundation of Liaoning Province (grant no. 201602291) and the Principal Foundation of Jinzhou Medical University (grant no. XZJJ20130217).

\section{References}

1. Kajstura J, Urbanek K, Perl S, Hosoda T, Zheng H, Ogórek B, Ferreira-Martins J, Goichberg P, Rondon-Clavo C, Sanada F, et al: Cardiomyogenesis in the adult human heart. Circ Res 107: 305-315, 2010.

2. Beltrami AP, Barlucchi L, Torella D, Baker M, Limana F, Chimenti S, Kasahara H, Rota M, Musso E, Urbanek K, et al: Adult cardiac stem cells are multipotent and support myocardial regeneration. Cell 114: 763-776, 2003.

3. Oh H, Bradfute SB, Gallardo TD, Nakamura T, Gaussin V, Mishina Y, Pocius J, Michael LH, Behringer RR, Garry DJ, et al: Cardiac progenitor cells from adult myocardium: Homing, differentiation, and fusion after infarction. Proc Natl Acad Sci USA 100: 12313-12318, 2003.

4. Bergmann O, Bhardwaj RD, Bernard S, Zdunek S, Barnabé-Heider F, Walsh S, Zupicich J, Alkass K, Buchholz BA, Druid $\mathrm{H}$, et al: Evidence for cardiomyocyte renewal in humans. Science 324: 98-102, 2009.

5. Bergmann O, Zdunek S, Alkass K, Druid H, Bernard S and Frisén J: Identification of cardiomyocyte nuclei and assessment of ploidy for the analysis of cell turnover. Exp Cell Res 317: 188-194, 2011.

6. Leri A, Quaini F, Kajstura J and Anversa P: Myocyte death and myocyte regeneration in the failing human heart. Ital Heart $\mathbf{J} 2$ (Suppl 3): 12S-14S, 2001.

7. Walsh S, Pontén A, Fleischmann BK and Jovinge S: Cardiomyocyte cell cycle control and growth estimation in vivo-an analysis based on cardiomyocyte nuclei. Cardiovasc Res 86: 365-373, 2010.

8. Kanawa M, Igarashi A, Ronald VS, Higashi Y, Kurihara H, Sugiyama M, Saskianti T, Pan H and Kato Y: Age-dependent decrease in the chondrogenic potential of human bone marrow mesenchymal stromal cells expanded with fibroblast growth factor-2. Cytotherapy 15: 1062-1072, 2013.

9. Portmann-Lanz CB, Schoeberlein A, Huber A, Sager R, Malek A, Holzgreve W and Surbek DV: Placental mesenchymal stem cells as potential autologous graft for pre- and perinatal neuroregeneration. Am J Obstet Gynecol 194: 664-673, 2006.

10. Fukuhara S, Tomita S, Yamashiro S, Morisaki T, Yutani C, Kitamura $S$ and Nakatani T: Direct cell-cell interaction of cardiomyocytes is key for bone marrow stromal cells to go into cardiac lineage in vitro. J Thorac Cardiovasc Surg 125: 1470-1480, 2003.

11. Rangappa S, Entwistle JW, Wechsler AS and Kresh JY: Cardiomyocyte-mediated contact programs human mesenchymal stem cells to express cardiogenic phenotype. J Thorac Cardiovasc Surg 126: 124-132, 2003.

12. Makino S, Fukuda K, Miyoshi S, Konishi F, Kodama H, Pan J, Sano M, Takahashi T, Hori S, Abe H, et al: Cardiomyocytes can be generated from marrow stromal cells in vitro. J Clin Invest 103: 697-705, 1999.

13. Valiunas V, Doronin S, Valiuniene L, Potapova I, Zuckerman J, Walcott B, Robinson RB, Rosen MR, Brink PR and Cohen IS: Human mesenchymal stem cells make cardiac connexins and form functional gap junctions. J Physiol 555: 617-626, 2004
14. Liu Y, Song J, Liu W, Wan Y, Chen X and Hu C: Growth and differentiation of rat bone marrow stromal cells: Does 5-azacytidine trigger their cardiomyogenic differentiation? Cardiovasc Res 58: 460-468, 2003.

15. Li K, Li SZ, Zhang YL and Wang XZ: The effects of dan-shen root on cardiomyogenic differentiation of human placenta-derived mesenchymal stem cells. Biochem Biophys Res Commun 415: 147-151, 2011.

16. Dominici M, Le Blanc K, Mueller I, Slaper-Cortenbach I, Marini F, Krause D, Deans R, Keating A, Prockop DJ and Horwitz E: Minimal criteria for defining multipotent mesenchymal stromal cells. The international society for cellular therapy position statement. Cytotherapy 8: 315-317, 2006.

17. Gojo S, Gojo N, Takeda Y, Mori T, Abe H, Kyo S, Hata J and Umezawa A: In vivo cardiovasculogenesis by direct injection of isolated adult mesenchymal stem cells. Exp Cell Res 288: 51-59, 2003.

18. Orlic D, Kajstura J, Chimenti S, Jakoniuk I, Anderson SM, Li B, Pickel J, McKay R, Nadal-Ginard B, Bodine DM, et al: Bone marrow cells regenerate infarcted myocardium. Nature 410: 701-705, 2001.

19. Murry CE, Soonpaa MH, Reinecke H, Nakajima H, Nakajima HO, Rubart M, Pasumarthi KB, Virag JI, Bartelmez SH, Poppa V, et al: Haematopoietic stem cells do not transdifferentiate into cardiac myocytes in myocardial infarcts. Nature 428: 664-668, 2004.

20. Li YG, Song L, Liu M, Hu ZB and Wang ZT: Advancement in analysis of Salviae miltiorrhizae Radix et Rhizoma (Danshen). J Chromatogr A 1216: 1941-1953, 2009.

21. The State Pharmacopoeia Commission of China: Chinese Pharmacopeia. Vol. 1. p76, 2015.

22. Li CQ, Xu WL, Shen ZG and Wang JZ: Comparison of the contents of 5 active components in Salvia miltiorrhiza from different habitats. Proceedings of Chemistry of Medicinal Plant and Analysis of Active Components of Traditional Chinese Medicine Seminar: 113-115, 2008.

23. Shang Q, Xu H and Huang L: Tanshinone IIA: A promising natural cardioprotective agent. Evid Based Complement Alternat Med 2012: 716459, 2012.

24. Jiang WD, Yu YZ, Liu WW, Chen YH, Wang YP and Huang TC: Effects of sodium tanshinone II-A sulfonate and propranolol on coronary collaterals in acutely infarcted dogs (author's transl). Zhongguo Yao Li Xue Bao 2: 29-33, 1981 (In Chinese).

25. Li CZ, Yang SC and Zhao FD: Effects of tanshinone II-A sulfonate on thrombus formation, platelet and blood coagulation in rats and mice. Zhongguo Yao Li Xue Bao 5: 39-42, 1984 (In Chinese).

26. Fan GW, Gao XM, Wang H, Zhu Y, Zhang J, Hu LM, Su YF, Kang LY and Zhang BL: The anti-inflammatory activities of Tanshinone IIA, an active component of TCM, are mediated by estrogen receptor activation and inhibition of iNOS. J Steroid Biochem Mol Biol 113: 275-280, 2009.

27. Kang YJ, Jin UH, Chang HW, Son JK, Lee SH, Son KH, Chang YC, Lee YC and Kim CH: Inhibition of microsomal triglyceride transfer protein expression and atherogenic risk factor apolipoprotein B100 secretion by tanshinone IIA in HepG2 cells. Phytother Res 22: 1640-1645, 2008.

28. Adams JD, Wang R, Yang J and Lien EJ: Preclinical and clinical examinations of Salvia miltiorrhiza and its tanshinones in ischemic conditions. Chin Med 1: 3, 2006.

29. Hardt SE and Sadoshima J: Glycogen synthase kinase-3beta: A novel regulator of cardiac hypertrophy and development. Circ Res 90: 1055-1063, 2002.

30. Cho J, Rameshwar P and Sadoshima J: Distinct roles of glycogen synthase kinase (GSK)-3alpha and GSK-3beta in mediating cardiomyocyte differentiation in murine bone marrow-derived mesenchymal stem cells. J Biol Chem 284: 36647-36658, 2009. 